\title{
Bound on the string tension by the excitation probability for a vortex
}

\author{
Tamás G. Kovács ${ }^{\mathrm{a}}$ and E. T. Tomboulis ${ }^{\mathrm{b}}$ * \\ anstituut-Lorentz for Theoretical Physics, University of Leiden, \\ P.O. Box 9506, NL-2300 RA Leiden, The Netherlands \\ b Physics Department, University of California, Los Angeles, CA 90095-1547
}

A lower bound on the string tension for large beta in SU(2) LGT is derived. The derivation is from first principles and bounds the string tension from below by the expectation for the excitation of a single 'tagged' thick vortex winding around the lattice. Thus confinement follows if this expectation remains nonvanishing at large beta. Numerical simulations are presented to show that this is indeed the case.

Over the last two years the center vortex picture of confinement has undergone substantial development by a series of numerical investigations as well as new analytical results (see e.g. [1] and references therein, and contributions to these proceedings). Here we report on a new analytical result relating the existence of nonzero string tension to the excitation probability of a vortex at weak coupling. The relation implies that nonvanishing expectation for an (arbitrarily) long vortex is a sufficient condition for confinement at weak coupling. We then present a measurement of this probability by numerical simulations.

We consider the Wilson loop $W[C]=\langle\operatorname{tr} U[C]\rangle$ in the $S U(2)$ LGT with plaquette action

$A_{p}(U)=\beta_{A}\left|\operatorname{tr} U_{p}\right|+\beta \operatorname{tr} U_{p}$,

and for large $\beta, \beta_{A}$. Our result is the following bound on the Wilson loop:

$W[C] \leq \exp (-\rho(\beta)|A|)$

where $|A|$ is the minimal loop area, and

$$
\begin{aligned}
\rho(\beta) & =(\text { Const }) \ln \left[1+\left\langle\theta_{\partial^{*} p}\right\rangle^{(+)} \tanh K_{0}\right] \\
& \approx(\text { Const }) e^{-4 \beta}\left\langle\theta_{\partial^{*} p}\right\rangle^{(+)} .
\end{aligned}
$$

with $K_{0} \equiv \frac{1}{2} \ln \operatorname{coth}(2 \beta)$.

The quantity $\left\langle\theta_{\partial^{*} p}\right\rangle^{(+)}$denotes the expectation of an operator creating magnetic flux forced to

\footnotetext{
${ }^{*}$ Talk presented by the last author at Lattice 99, Pisa,
} Italy. wind completely around the (periodic) lattice. It is defined as follows.

Define for a 3-cube $c$ :

$\eta_{c}=\prod_{p \in \partial c} \operatorname{sign} \operatorname{tr} U_{p}$.

A configuration such that $\eta_{c}=-1$ represents a Dirac monopole of $Z(2)$ flux residing on the cube c. The set of cubes on which $\eta_{c}=-1$ must form coclosed sets, i.e. closed sets on the dual lattice (Bianchi identity - magnetic current conservation). Thus in $d=4$ (where a cube is dual to a bond), a coclosed set of cubes is a closed loop of dual bonds: a $Z(2)$ monopole loop. The smallest such set is the coboundary of a plaquette (figure 1).

By virtue of the conservation constraint, the presence (absence) of a minimal length monopole 'loop' on the coboundary $\partial^{*} p$ of a plaquette $p$ is characterized by $\theta_{\partial^{*} p}=1\left(\theta_{\partial^{*} p}=0\right)$, where

$\theta_{\partial^{*} p} \equiv \prod_{c \in \partial^{*} p} \frac{1}{2}\left[1-\eta_{c}\right]$

It is the expectation $\left\langle\theta_{\partial^{*} p}\right\rangle^{(+)}$of this quantity (5), for some fixed plaquette $p$, that occurs in (3). The choice of $p$ is irrelevant by translational invariance. The ' + ' in \langle\rangle$^{(+)}$signifies that the expectation of $\theta_{\partial^{*} p}$, is computed with:

(i) plaquette action: $\left(\beta_{A}+\beta\right)\left|\operatorname{tr} U_{p}\right|$; 


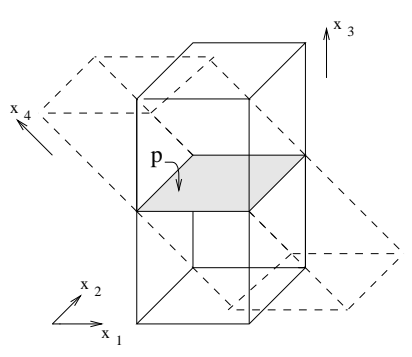

$\Lambda$

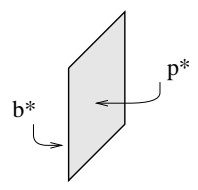

$\Lambda *$

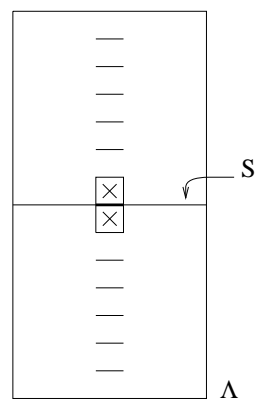

Figure 1. Coboundary of plaquette $p$ consists of the set of cubes having the plaquette (shaded) on their boundary. On the dual lattice, this corresponds to the boundary of the dual plaquette $p^{*}(\mathrm{~d}=4)$. The smallest monopole loop has $\eta_{c}=-1$ on the cubes of such a single plaquette coboundary.

(ii) $\eta_{c}=1$ for all 3 -cubes on the lattice other than the cubes belonging to $\partial^{*} p$ in the numerator, and for all 3-cubes in the denominator (i.e. the partition function) of the expectation;

(iii) $\operatorname{sign} U_{p^{\prime}}=1$ for every plaquette $p^{\prime}$ on the 2-dimensional plane $S$ spanning the lattice and containing the plaquette $p$ of $\partial^{*} p$.

It is important to note, and easily seen that, with periodic boundary conditions, (i)-(iii) imply that $\left\langle\theta_{\partial^{*} p}\right\rangle^{(+)}$depends only on coset $S U(2) / Z(2) \sim$ $S O(3)$ rather than $S U(2)$ bond variable configurations.

The expectation $\left\langle\theta_{\partial^{*} p}\right\rangle^{(+)}$is now seen to have the following physical meaning. (We refer to [2], [3] for a physical discussion of vortices and constraints on fluxes on the lattice.) Constraint (iii) forbids any net vortex flux from crossing the surface $S$. Furthermore, monopole excitation is forbidden everywhere by constraint (ii) except on the coboundary of the plaquette $p$ on $S$, where the factor $\theta_{\partial^{*} p}$ enforces the presence of a monopole loop. The Dirac sheet in the $S O(3)$ configuration, representing the vortex flux attached to this monopole loop, is thus forced to wind around the periodic lattice in the perpendicular directions.
Figure 2. Tagged vortex winding around the lattice.

$\left\langle\theta_{\partial^{*} p}\right\rangle^{(+)}$is then the excitation probability amplitude for a vortex completely winding around the lattice in the directions perpendicular to the surface $S$ 'anchored' by a minimal-length monopole loop, encircing $S$ (figure 2). This is in fact a version of a 't Hooft loop operator [4]. Shrinking the monopole loop to a point, would result in the flux of a complete, 'unpunctured' vortex trapped inside the periodic lattice.

Eqs. (2)-(3) then imply nonvanishing string tension provided the expectation $\left\langle\theta_{\partial^{*} p}\right\rangle^{(+)}$remains nonvanishing in the large volume limit at (arbitrarily) large $\beta$. What decides this is whether the free-energy cost of the flux forced to wind around the (periodic) lattice remains finite in the large lattice limit. This can happen if flux can efficiently spread out in the transverse directions to compensate for the cost along the winding directions [2], [3]. The monopole loop itself represents a local effect of fixed action cost, and serves to 'anchor' and tag the vortex. This is a very convenient device that leads to derivation of (2)-(3).

Indeed, the derivation relies on a factorization inequality for the expectation of $n$ tagged vortices in terms of the product of the expectation of $(n-1)$ tagged vortices times that of a single tagged vortex. It further uses the $S O(3) \times Z(2)$ formulation of the $S U(2)$ LGT [2], and subsequent duality transformation on the $Z(2)$ part. The somewhat lengthy details will appear elsewhere. 
The finite local cost associated with the monopole loop site can in fact be explicitly extracted, and then $\left\langle\theta_{\partial^{*} p}\right\rangle^{(+)}$can be related to the expectation for a single unpunctured vortex winding around the lattice, i.e. essentially 't Hooft's magnetic-flux free-energy order parameter [4]. Now both the latter and $\left\langle\theta_{\partial^{*} p}\right\rangle^{(+)}$are nonvanishing in the large volume limit already when evaluated in the semiclassical approximation for spacetime dimension $d \leq 4$. One expects this to persist and in fact be improved in the full theory where flux can spread out nonperturbatively. In the absence of an analytical proof, we have resorted to numerical evaluation of the vortex excitation expectation (magnetic flux freeenergy).

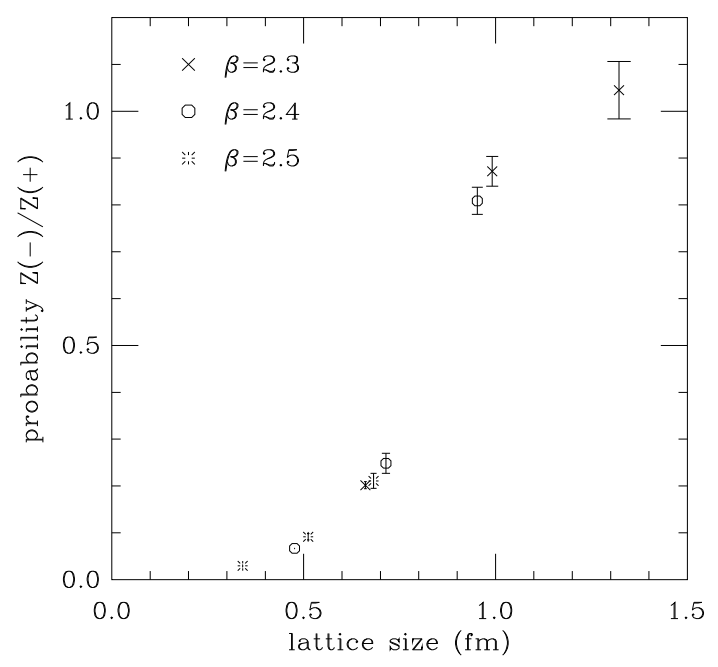

Figure 3. Vortex probability (magnetic-flux free-energy) vs. lattice size

Measurement was performed by the multihistogram method [5. The method tends to be computationally expensive. It was used in [6] to compute the free energy of a $\mathrm{Z}(2)$ monopole pair as a function of the pair's separation. The result of our computation is shown in figure 3. The lattice spacings are $a=0.119 \mathrm{fm}$ and $a=0.085 \mathrm{fm}$ for $\beta=2.4$ and $\beta=2.5$, respectively. As expected by physical reasoning, not only does the vortex free energy cost remain finite as the lattice volume grows, but it tends to zero, i.e. the weighted probability for the presence of a vortex goes to unity for sufficiently large lattice. This reflects the exponential spreading of color-magnetic flux in a confining phase.

In conclusion, we have seen that nonvanishing expectation for an arbitrarily long spread-out vortex is sufficient to ensure that the $S U(2)$ LGT remains in a confining phase at any large $\beta$. This should be combined with the result that the presence of vortices at weak coupling is also necessary for confinement: constraining a Wilson loop to be insensitive to the presence of thick vortices linking with it leads to nonconfining behavior [7]. Both these statements are clearly demonstrated in the numerical simulations. In fact, the measurement of the magnetic-flux free-energy presented here is interesting in its own right as it also shows that the probability at large $\beta$ for the presence in the vacuum of a sufficiently spread-out vortex actually tends to one. This is in complete agreement with the results of the closely connected measurements in $[6]$.

This work was supported by FOM, and NSF grant nsf-phy 9531023 .

\section{REFERENCES}

1. C.T.H. Davies et al (eds), Lattice 97 Proceedings (Edinburgh), Nucl. Phys. B (Proc. Suppl.) 63 (1998); T. DeGrand et al (eds), Lattice 98 Proceedings (Boulder), Nuc. Phys. B (Proc. Supp.) 73 (1999).

2. T.G. Kovács and E.T. Tomboulis, Phys. Rev. D57 (1998) 4054.

3. L. Yaffe, Phys. Rev. D21 (1979) 1574.

4. G. 't Hooft, Nuc. Phys. B138 (1978) 1; ibid B153 (1979) 141.

5. A. Ferrenberg and R. Swendsen, Phys. Rev. Lett. 63 (1989) 1195.

6. C. Hoelbing, C. Rebbi, and V.A. Rubakov, Nuc. Phys. B (Proc. Supp.) 73 (1999) 527, hep-lat/9809113.

7. T.G. Kovács and E.T. Tomboulis, Journal of Math. Phys. (in press), hep-lat/9806030. 